\title{
Saffron carotenoids inhibit STAT3 activation and promote apoptotic progression in IL-6-stimulated liver cancer cells
}

\author{
BUYUN KIM and BYOUNGDUCK PARK \\ College of Pharmacy, Keimyung University, Dalseo-Gu, Daegu 704-701, Republic of Korea
}

Received August 17, 2017; Accepted January 23, 2018

DOI: $10.3892 /$ or.2018.6232

\begin{abstract}
Signal transducer and activator of transcription 3 (STAT3) is involved in the survival, proliferation, angiogenesis, invasion and metastasis of tumor cells. In addition, interleukin-6 (IL-6) has been reported to be closely related to STAT3 activity. In the present study, we investigated whether crocin, a major glycosylated carotenoid derived from saffron, can modulate the IL-6/STAT3 pathway to induce growth inhibition and sensitivity to cancer cell apoptosis. We determined that crocin inhibited STAT3 activation induced by IL-6 in hepatocellular carcinoma Hep3B and HepG2 cells. STAT3 suppression was mediated through the inactivation of Janus kinase 1/2(JAK1, JAK2) and Src kinase in both liver cancer cell lines. Furthermore, crocin induced the expression of protein tyrosine phosphatase (PTP) SHP-1, which led to STAT3 dephosphorylation. Deletion of the SHP-1 gene by siRNA recovered the inhibitory effects of crocin, suggesting an important role for SHP-1. Moreover, crocin downregulated the expression of STAT3-regulated anti-apoptotic (Bcl-2, survivin), proliferative (cyclin D1), invasive (CXCR4) and angiogenic (VEGF) proteins. Conversely, crocin increased the pro-apoptotic (BAX) protein, which was correlated with the induction of apoptosis and inhibition of proliferation. Overall, these results provide evidence that crocin has the potential for anticancer activity through inhibition of the IL-6/STAT3 signaling pathway, especially in liver cancer.
\end{abstract}

\section{Introduction}

Hepatocellular carcinoma (HCC) represents approximately $90 \%$ of primary liver cancers and is the second leading cause of mortality among all human malignancies (1). Unfortunately, patients often present with advanced HCC that is unresectable, recurrent or metastatic. Systemic chemotherapy is ineffective

Correspondence to: Professor Byoungduck Park, College of Pharmacy, Keimyung University, 1095 Dalgubeoldaero, Dalseo-Gu, Daegu 704-701, Republic of Korea

E-mail: bdpark@kmu.ac.kr

Key words: saffron carotenoids, crocin, IL-6, STAT3, SHP-1, liver cancer, apoptosis in these patients (2). Classical radiation therapy is not a standard adjuvant for patients with HCC due to intolerance and resistance of the liver to radiation. In this aspect, developing effective therapies for liver cancer is one of the most challenging goals in cancer research. There have been many studies that have revealed that local expression of interleukin-6 (IL-6) in the liver was increased in patients with chronic hepatitis. Moreover, signal transducer and activator of transcription 3 (STAT3) plays a major role in the pathogenesis of hepatocellular carcinoma (3). IL-6 is a secreted multifunctional cytokine that has been identified as a master regulator of the STAT3 signaling pathway $(4,5)$. Furthermore, tumor-associated macrophages were revealed to activate IL-6/STAT3 signaling in neighboring HCC stem cells via secretion of IL-6 in the HCC microenvironment and consequently promote tumor progression. Numerous studies have demonstrated that STAT3 participates in regulating gene expression related to cell proliferation (c-myc, cyclin D1), survival (Mcl-1, Bcl-xL and survivin), invasion (matrix metalloproteinase-9), and angiogenesis (VEGF) (6). Activation of STAT3 was also associated with decreased survival rates in HCC (7) and other types of cancer. These findings indicate that STAT3 has emerged as a therapeutic target for the development of anticancer agents.

Thus far, the use of anticancer drugs derived from traditional medicine offers new opportunities to improve existing standard treatments for cancer and other diseases. Crocus sativus L. (saffron) has been known as a flavoring agent, food coloring and traditional herbal medicine. Major components including safranal, picrocrocin, crocetin and crocin are considered as the pharmacologically active components of saffron (8). Crocin [digentiobiosyl all-trans-crocetin (8,8'-di-apocarotene$8,8^{\prime}$-dioic acid) ester] is a major glycosylated carotenoid found in saffron (9) that has various pharmacological effects such as antioxidant $(10,11)$, anti-atherosclerotic (12), antidepressant (13) and anti-inflammatory activities $(14,15)$. Moreover, some studies have shown anticancer activities of crocin against human leukemia (16), colorectal (17), breast (18) and bladder cancer cell lines (19). In addition, crocin is a potential nutraceutical for protecting liver tissue from hepatic steatosis (20). Based on these studies, it is expected that crocin could possibly be used to prevent and treat various cancers in the near future. However, the direct influence of crocin on the STAT3 signaling pathway has not been investigated. Thus, we examined whether crocin exerts therapeutic effects by modulating the IL-6/STAT3 cell signaling pathways. 
In the present study, we revealed that crocin suppressed the activation of the STAT3 pathway in IL-6-stimulated hepatocellular carcinoma cells. Crocin suppressed the activation of non-receptor protein tyrosine kinases (JAK1, JAK2 and $\mathrm{Src}$ ) and upregulated $\mathrm{SH} 2$-containing tyrosine phosphatase (SHP-1). Crocin also downregulated STAT3-regulated gene products and promoted apoptotic progression in liver cancer cells.

\section{Materials and methods}

Reagents. Crocin, with the chemical structure presented in Fig. 1A, was provided by Dr Ki Yong Lee (College of Pharmacy, Korea University). A $10-\mathrm{mM}$ solution of crocin was prepared in dimethyl sulfoxide (DMSO), stored as small aliquots at $-20^{\circ} \mathrm{C}$, and then diluted as needed in cell culture medium. RPMI-1640, fetal bovine serum (FBS), and an antibiotic-antimycotic mixture were obtained from Gibco-BRL (Grand Island, NY, USA). The antibodies used were as follows: p-STAT3 (1:1,000; rabbit, monoclonal, cat. no. 9145), STAT3 (1:1,000; rabbit, monoclonal, cat. no. 12640), p-JAK1 (1:1,000; rabbit, polyclonal, cat. no. 3331), p-JAK2 (1:1,000; rabbit, monoclonal, cat. no. 8082), p-Src (1:1,000; rabbit, polyclonal, cat. no. 2101), Src (1:1,000; rabbit, polyclonal, cat. no. 2108), SHP-1 (1:1,000; rabbit, monoclonal, cat. no. 3759), cleaved PARP (1:1,000; rabbit, monoclonal, cat. no. 5625), cleaved caspase-9 (1:1,000; rabbit, monoclonal, cat. no. 7237), cleaved caspase-3 (1:1,000; rabbit, polyclonal, cat. no. 9661), $\beta$-actin (1:1,000; rabbit, monoclonal, cat. no. 4970) and anti-rabbit IgG (1:5,000; rabbit, polyclonal, cat. no. 14708) were obtained from Cell Signaling Technology Inc. (Danvers, MA, USA). Cyclin D1 (1:1,000; rabbit polyclonal, cat. no. sc-718), VEGF (1:1,000; rabbit, polyclonal, cat. no. sc-152), JAK1 (1:1,000; rabbit, polyclonal, cat. no. sc-277), JAK2 (1:1,000; rabbit, polyclonal, cat. no. sc-278), Bax (1:1,000; rabbit, polyclonal, cat. no. sc-493), Bcl-2 (1:1,000; rabbit, polyclonal, cat. no. sc-492) and goat anti-mouse $\operatorname{IgG}(1: 5,000$; mouse, monoclonal, cat. no. sc-2355) were purchased from Santa Cruz Biotechnology (Santa Cruz, CA, USA). CXCR4 (1:10,000; rabbit, polyclonal, cat. no. sc-492) was obtained from Abcam (Cambridge, MA, USA). Recombinant human IL-6 was purchased from Invitrogen (Groningen, The Netherlands). The siRNA for SHP-1 was obtained from Ambion (Austin, TX, USA). Control siRNA was obtained from Santa Cruz Biotechnology (Santa Cruz, CA, USA).

Cell cultures. Human hepatocellular carcinoma cells (Hep3B, HepG2), human colon cancer cells (HCT116) and human breast cancer cells (MDA-MB-231) were cultured in Dulbecco's modified Eagle's medium (DMEM) supplemented with 10\% FBS and $1 \%$ antibiotics. Human pancreatic cancer cells (BxPC3), human lung cancer cells (A549) and human ovarian cancer cells (A2780) were grown in RPMI-1640 (Gibco Laboratories, Grand Island, NY, USA) supplemented with 5\% FBS and 1\% antibiotics. Cells were maintained at $37^{\circ} \mathrm{C}$ in an atmosphere of $5 \% \mathrm{CO}_{2}-95 \%$ air. All cells were passaged at $80 \%$ confluence in $0.25 \%$ trypsin-EDTA for 3-5 min.

Western blotting. The whole-cell extracts were lysed with RIPA buffer (20 mM Tris-HCl, pH 7.5, $150 \mathrm{mM} \mathrm{NaCl}, 1 \mathrm{mM}$
EDTA, $50 \mathrm{mM} \beta$-glycerophosphate, $1 \% \mathrm{NP}-40,1 \mathrm{mM} \mathrm{Na}_{3} \mathrm{VO}_{4}$ and $1 \mathrm{x}$ protease inhibitor cocktail). The extracted proteins were then resolved on sodium dodecyl sulphate-polyacrylamide gel electrophoresis (SDS-PAGE). After electrophoresis, the proteins were electrotransferred to polyvinylidene fluoride (PVDF) membranes, blocked with 5\% non-fat milk. The blots were subjected to a standard immune detection procedure using specific antibodies overnight at $4^{\circ} \mathrm{C}$. The blot was washed, exposed to HRP-conjugated secondary antibodies for $1 \mathrm{~h}$, and finally examined by enhanced chemiluminescence reaction using ECL reagents (Amersham Pharmacia Biotech, Buckinghamshire, UK).

The primary antibodies used were as follows: p-STAT3 (1:1,000; rabbit, monoclonal; cat. no. 9145), STAT3 (1:1,000; rabbit, monoclonal; cat. no. 12640), p-JAK1 (1:1,000; rabbit, polyclonal; cat. no. 3331), p-JAK2 (1:1,000; rabbit, monoclonal; cat. no. 8082), p-Src (1:1,000; rabbit, polyclonal; cat. no. 2101), Src (1:1,000; rabbit, polyclonal; cat. no. 2108), SHP-1 (1:1,000; rabbit,monoclonal; cat.no.3759), cleaved PARP (1:1,000; rabbit, monoclonal; cat. no. 5625), cleaved caspase-9 $(1: 1,000$; rabbit, monoclonal; cat. no. 7237), cleaved caspase-3 (1:1,000; rabbit, polyclonal; cat. no. 9661), $\beta$-actin (1:1,000; rabbit, monoclonal; cat. no. 4970; all from Cell Signaling Technology, Inc.), cyclin D1 (1:1,000; rabbit, polyclonal; cat. no. sc-718), VEGF (1:1,000; rabbit, polyclonal; cat. no. sc-152), JAK1 (1:1,000; rabbit, polyclonal; cat. no. sc-277), JAK2 (1:1,000; rabbit, polyclonal; cat. no. sc-278), Bax (1:1,000; rabbit, polyclonal; cat. no. sc-493), Bcl-2 (1:1,000; rabbit, polyclonal; cat. no. sc-492; all from Santa Cruz Biotechnology), CXCR4 (1:10,000; rabbit, polyclonal; cat. no. sc-492; Abcam). The secondary antibodies used were goat anti-mouse $\operatorname{IgG}$ (1:5,000; mouse, monoclonal; cat. no. sc-2355; Santa Cruz Biotechnology) and anti-rabbit $\operatorname{IgG}(1: 5,000$; rabbit, polyclonal; cat. no. 14708; Cell Signaling Technology, Inc.).

To detect the expression of STAT3-regulated proteins, Hep3B and HepG2 cells were treated with $20 \mu \mathrm{M}$ crocin for the indicated time-points. The cells were then washed and extracted by incubation for $30 \mathrm{~min}$ on ice in RIPA buffer. The lysate was centrifuged, and the supernatant was collected. Whole-cell protein extract $(20 \mu \mathrm{g})$ was resolved on 10-12\% SDS-PAGE and then electro-transferred onto a PVDF membrane. Proteins were detected by incubation with primary antibodies against CXCR4, SHP-1, p-Src, Src, p-JAK1, JAK1, p-JAK2, JAK2, VEGF, survivin, Bax, Bcl-2, cyclin D1, caspase-9, caspase-3, PARP and $\beta$-actin, followed by secondary antibodies conjugated with horseradish peroxidase. Immuno-complexes were visualized by a chemiluminescence reaction using ECL reagents. Signal intensity was quantified by an image analyzer (LAS 4000).

Preparation of cytosolic and nuclear fraction. Hep3B and HepG2 cells were treated with $10 \mathrm{ng} / \mathrm{ml}$ IL- 6 for $1 \mathrm{~h}$, and then both cell lines were washed two times and scraped with PBS. The cell suspension was incubated for $5 \mathrm{~min}$ on ice with buffer A (10 mM HEPES-KOH pH 7.9, $1.5 \mathrm{~mm} \mathrm{MgCl}_{2}, 10 \mathrm{mM} \mathrm{KCl}$, $0.5 \mathrm{mM}$ DTT, $300 \mathrm{mM}$ saccharose, $0.1 \% \mathrm{NP}-40$ and $0.5 \mathrm{mM}$ PMSF) and centrifuged for $5 \mathrm{~min}$ at $1,000 \mathrm{xg}\left(4^{\circ} \mathrm{C}\right)$. The supernatants (cytosol fraction) were picked up and the cell pellets were suspended and incubated for $10 \mathrm{~min}\left(4^{\circ} \mathrm{C}\right)$ in buffer B (20 mM HEPES-KOH pH 7.9, 20\% glycerol, $100 \mathrm{mM} \mathrm{KCl,}$ 
A

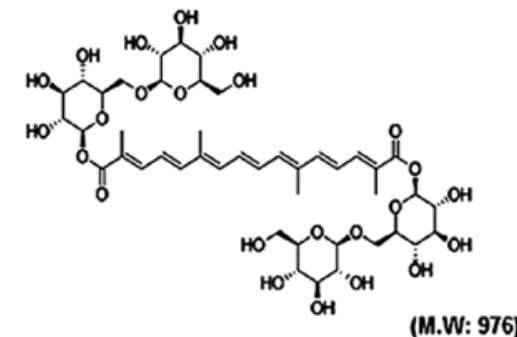

B

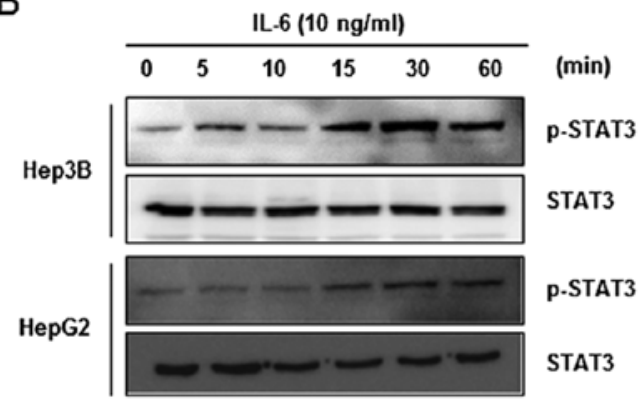

C

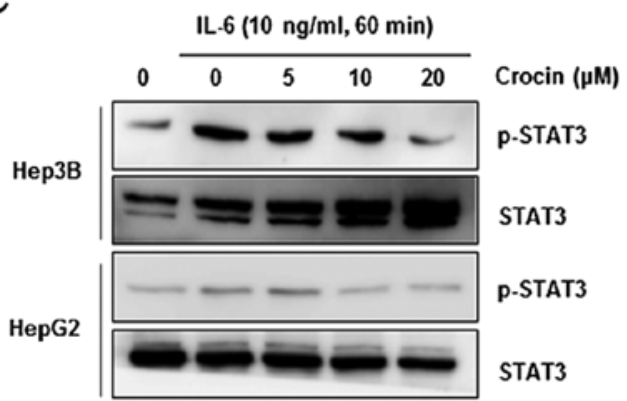

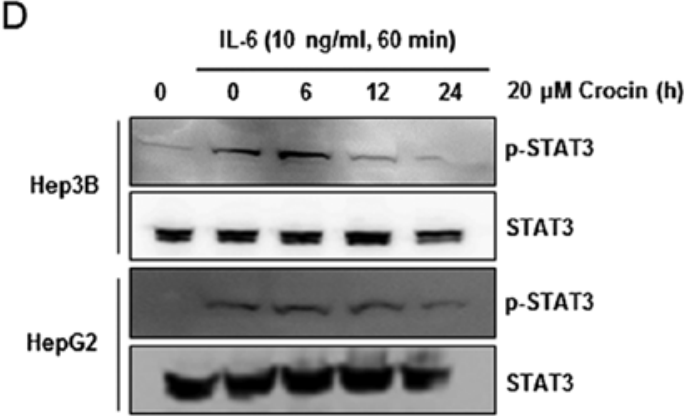

$\mathrm{E}$

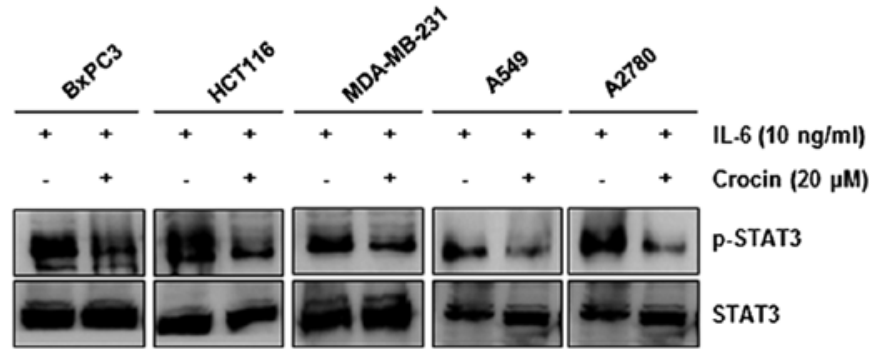

Figure 1. Crocin inhibits both inducible and constitutive STAT3 activation in liver cancer cells. (A) Chemical structure of crocin. (B) IL-6 induced STAT3 activity in liver cancer cells. Hep3B and HepG2 cells $\left(1 \times 10^{6} / \mathrm{ml}\right)$ were treated with IL-6 (10 ng/ml) for indicated time-points. Whole-cell extracts were prepared, and phosphorylated STAT3 was detected by western blotting as described in Materials and methods. (C) Crocin downregulated IL-6-induced phospho-STAT3 in a dose-dependent manner. Hep3B and HepG2 cells $\left(1 \times 10^{6} / \mathrm{ml}\right)$ were treated with the indicated concentrations of crocin for $24 \mathrm{~h}$ and then stimulated with IL-6 (10 ng/ml) for $60 \mathrm{~min}$. Whole-cell extracts were prepared, and phospho-STAT3 was detected by western blotting. The same blots were stripped and reprobed with the STAT3 antibody to verify equal protein loading. (D) Crocin downregulated IL-6-induced phospho-STAT3 in a time-dependent manner. Hep3B and HepG2 cells $\left(1 \times 10^{6} / \mathrm{ml}\right)$ were treated with $20 \mu \mathrm{M}$ crocin for the indicated time-points and then stimulated with IL-6 (10 ng/ml) for $60 \mathrm{~min}$. Whole-cell extracts were prepared, and phospho-STAT3 was detected by western blotting. (E) Crocin suppresses phospho-STAT3 levels in breast and colon cancer cells. MDA-MB-231, HCT116, BxPC3, A549 and A2780 cells $\left(1 \times 10^{6} / \mathrm{ml}\right)$ were not or were treated with $20 \mu \mathrm{M}$ of crocin and stimulated with IL-6 (10 ng/ml) for $24 \mathrm{~h}$, after which whole-cell extracts were prepared, and phospho-STAT3 was detected by western blotting. The same blots were stripped and reprobed with the STAT3 antibody.

$100 \mathrm{mM} \mathrm{NaCl}, 0.2 \mathrm{mM}$ EDTA, $0.5 \mathrm{mM}$ PMSF and $0.5 \mathrm{mM}$ DTT). These were immediately centrifuged at $17,000 \mathrm{xg}$ for $5 \mathrm{~min}\left(4^{\circ} \mathrm{C}\right)$ and the supernatants (nuclear fraction) were recovered.

Electrophoretic mobility shift assay (EMSA). Hep3B and HepG2 cells were grown to $\sim 80 \%$ confluence and nuclear protein was prepared. STAT3/DNA-binding activity was detected by EMSA using the DIG Gel Shift kit (Roche, Mannheim, Germany) according to the manufacturer's protocol. Briefly, the nuclear proteins were subjected to hybridization with a double-stranded, DIG-labelled oligonucleotide probe containing the consensus binding site for
STAT3 (5-CTTCATTTCCCGTAAATCCCTAAAGCT-3 and 5-AGCTTTAGGGATTTACGGGAAATGA-3).

Immuno fluorescence for STAT3 translocation. Crocin-treated cells were plated on a glass slide and fixed in $3.7 \%$ formaldehyde in PBS. The membrane was permeabilized by treating cells for 5 min with $0.1 \%$ Triton X-100 in PBS. After a brief washing in PBS, the slides were blocked with 5\% bovine serum albumin for $1 \mathrm{~h}$ and then incubated with anti-human STAT3 antibody (1:1,000; rabbit, monoclonal, cat. no. 9145; Cell Signaling Technology, Inc.) for $1 \mathrm{~h}$ at room temperature. After being washed, the slides were incubated with the secondary antibody Alexa Flour 488 (1:100; goat anti-rabbit IgG, 
A

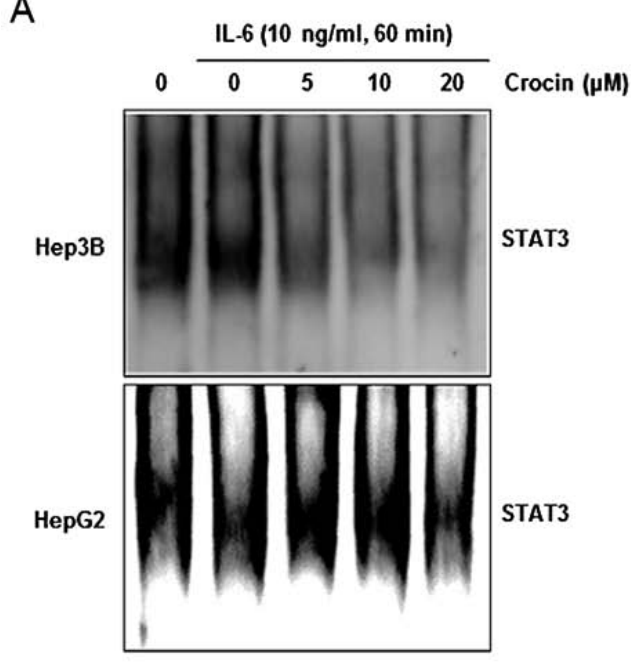

C

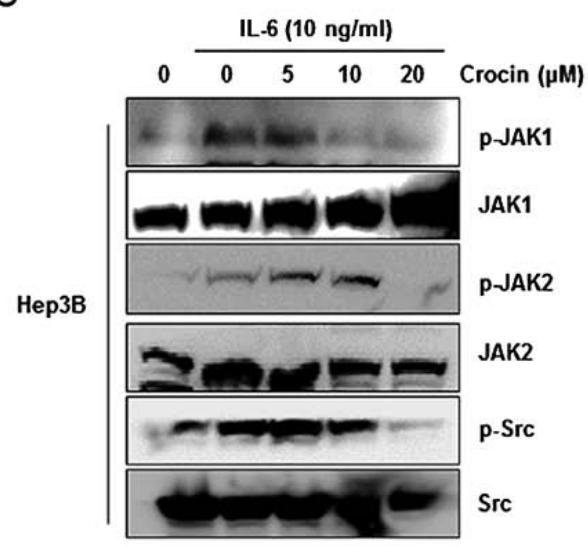

B
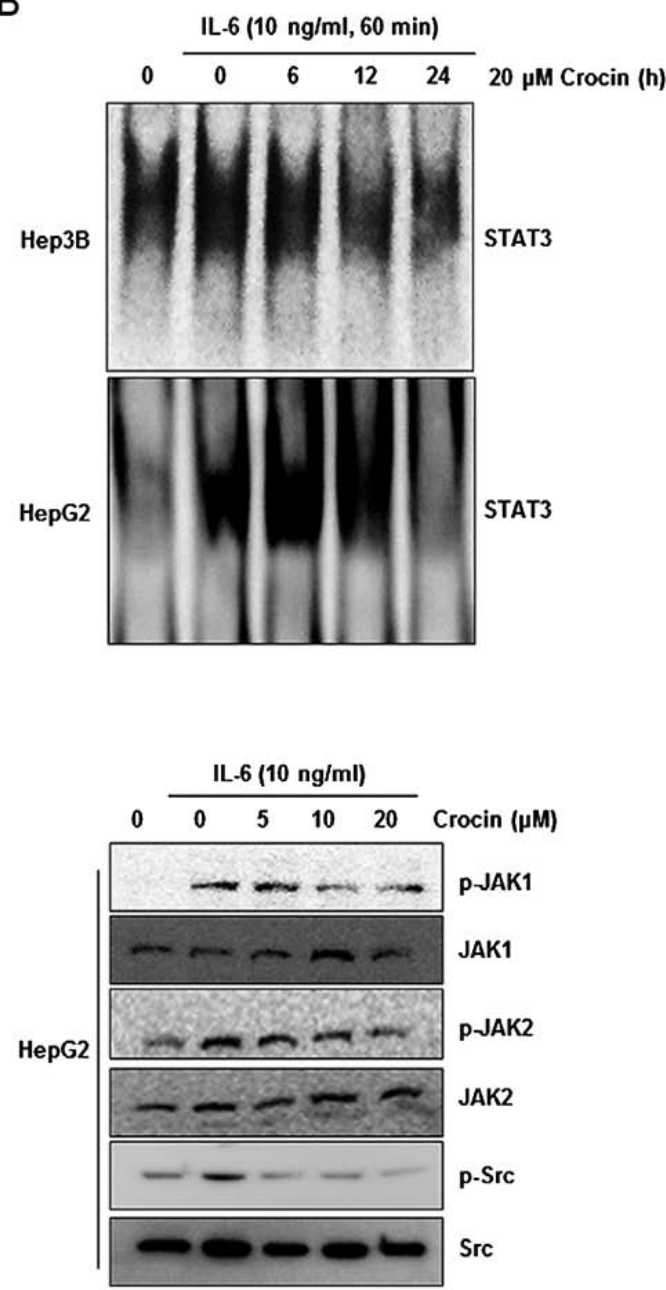

Figure 2. Crocin inhibits DNA-binding activity of STAT3 in IL-6-stimulated liver cancer cells. (A) Hep3B and HepG2 cells (1x10 $/ \mathrm{ml})$ were treated with the indicated concentrations of crocin for $24 \mathrm{~h}$ and then stimulated with IL-6 $(10 \mathrm{ng} / \mathrm{ml})$ for $60 \mathrm{~min}$. In addition, nuclear STAT3 levels were analyzed using EMSA as described in Materials and methods. (B) Hep3B and HepG2 cells $\left(1 \times 10^{6} / \mathrm{ml}\right)$ were treated at the indicated time-pointswith $20 \mu \mathrm{M}$ crocin and then stimulated with IL-6 $(10 \mathrm{ng} / \mathrm{ml})$ for $60 \mathrm{~min}$. In addition nuclear STAT3 levels were analyzed using EMSA. (C) Crocin inhibits IL-6-induced JAK1, JAK2, and Src kinase activation in Hep3B and HepG2 cells. These cells were treated with the indicated concentrations of crocin for $24 \mathrm{~h}$ and then stimulated with IL-6 $(10 \mathrm{ng} / \mathrm{ml})$ for $60 \mathrm{~min}$. Whole-cell extracts were prepared and $40 \mu \mathrm{g}$ aliquots of those extracts were resolved on $10 \%$ SDS-PAGE, electro transferred onto PVDF membranes and probed with p-JAK1, p-JAK2 and p-Src. The same blots were stripped and reprobed with JAK1, JAK2 and Src antibodies to ascertain equal protein loading.

polyclonal; cat. no. A11008; Thermo Fisher Scientific, Inc., Carlsbad, CA, USA) for $30 \mathrm{~min}$ and counterstained for nuclei with Hoechst 33342 (ImmunoChemistry Technologies, LLC, Bloomington, MN, USA) for 10 min. The slides were mounted using ProLong ${ }^{\circledR}$ Gold Antifade Mountant reagent (Molecular Probes $^{\circledR}$ by Life Technologies ${ }^{\mathrm{TM}}$; Thermo Fisher Scientific, Inc.). Fluorescence micrographs were acquired with a fluorescence microscope (Nikon ECLIPSE Ti-U; Nikon Corporation, Tokyo, Japan).

Transfection with SHP-1 siRNA. Human hepatocellular carcinoma cells (Hep3B) were plated in 6-well plates and allowed to adhere for $24 \mathrm{~h}$. On the day of transfection, $9 \mu \mathrm{l}$ of Lipofectamine RNAiMAX transfection reagent (Invitrogen; Thermo Fisher Scientific, Inc.) was added to $50 \mathrm{~nm}$ SHP-1 siRNA (sense-GGGCAAGAACCGCUACAAGtt, antisenseCUUGUAGCGGUUCUUGCCCtt) (Ambion) in a final volume of $150 \mu 1$ of culture medium. After $48 \mathrm{~h}$ of transfection, the cells were treated with crocin for $24 \mathrm{~h}$, and whole-cell extracts were prepared for SHP-1, STAT3, and phospho-STAT3 analysis by western blotting.

TUNEL assay. The in situ labeling of apoptotic cells was performed using a terminal deoxynucleotidyl transferase (TdT)-mediated deoxyuridine triphosphate-digoxigenin (dUTP) nick-end labeling (TUNEL) assay with commercial kits (Roche, Mannheim, Germany).

Statistical analysis. The data are presented as the mean values \pm standard error (SE) of the mean of at least three separate experiments. Comparisons were made using Student's t-test. For all analysis, a two-sided P-value $<0.05$ was considered to indicate statistical significance.

\section{Results}

Crocin inhibits inducible STAT3 activation in IL-6-stimulated cancer cells. Since previous research has shown that STAT3 
can be phosphorylated at tyrosine residue 705 by stimulation with cytokines (IL-6 and IL-11) and growth factors (EGF and PDGF) $(4,21)$, we first investigated whether IL-6 induced STAT3 phosphorylation in hepatocellular carcinoma HepG2 and Hep3B cells. The results revealed that IL- 6 induced STAT3 phosphorylation after as few as $15 \mathrm{~min}$ and increased its phosphorylation for up to $60 \mathrm{~min}$ (Fig. 1B) in both cell lines. Then, we investigated whether crocin modulated IL-6-induced STAT3 phosphorylation. HepG2 and Hep3B cells were incubated with different concentrations of crocin for $24 \mathrm{~h}$ and then treated with IL- 6 for 60 min. Whole-cell extracts were prepared and assessed for STAT3 phosphorylation by western blot analysis using an antibody that recognizes STAT3 phosphorylated at tyrosine 705. Crocin inhibited IL-6-induced phosphorylation of STAT3 in Hep3B and HepG2 cells, with maximum inhibition occurring at $20 \mu \mathrm{M}$ (Fig. 1C). When we examined the incubation time required for crocin to suppress STAT3 activation in both cell lines, inhibition of STAT3 phosphorylation was revealed to be in a time-dependent manner, with maximum inhibition occurring at $24 \mathrm{~h}$ (Fig. 1D). Persistent or inducible activation of STAT3 has been frequently found in the majority of human cancers including pancreatic (22), lung (23), ovarian (24), breast (25) and colorectal (26) cancers. Thus, we investigated whether crocin inhibited STAT3 activation in IL-6-stimulated various cancer cells. Our data revealed that crocin suppressed inducible STAT3 activation in HCT116 and MDA-MB-231 cell lines as well as in BxPC3, A549 and A2780 cells (Fig. 1E), and these findings revealed that inhibition of STAT3 activation by crocin was not cell type-specific.

Crocin inhibits the DNA-binding activity of STAT3 in IL-6-stimulated liver cancer cells. Phosphorylation of STAT3 at Tyr705 leads to dimerization, nuclear translocation, where it binds to STAT3-specific DNA-binding elements and regulates gene transcription (27). We therefore determined whether crocin suppressed the DNA-binding activity of STAT3. Cells were treated with different concentrations of crocin for $24 \mathrm{~h}$ or $20 \mu \mathrm{M}$ crocin at different time-points. Crocin inhibited STAT3-DNA binding in IL-6-stimulated Hep3B and HepG2 cells in a dose- and time-dependent manner (Fig. 2A and B).

Crocin inhibits JAK1, JAK2, and Src kinase activation in IL-6-stimulated liver cancer cells. The tyrosine residues in the STAT protein are phosphorylated by a variety of upstream signaling kinases including JAKs and Src (28). The effect of crocin on the activation of JAK1, JAK2 and Src in IL-6-stimulated Hep3B and HepG2 cells was assessed, since the inhibitory effect of crocin on STAT3 phosphorylation was due to suppression of the upstream signaling pathway. The results revealed that JAK1, JAK2 and Src were activated by IL-6, and treatment with crocin inhibited the phosphorylation of these proteins in a dose-dependent manner (Fig. 2C).

Crocin depletes the nuclear pool of STAT3 in IL-6-stimulated liver cancer cells. Since it is unclear that phosphorylation is essential for nuclear transfer and carcinogenic functions of STAT3 (27), we investigated whether crocin suppresses nuclear translocation of STAT3. As shown in Fig. 3, crocin inhibited the translocation of STAT3 to the nucleus in IL-6-stimulated
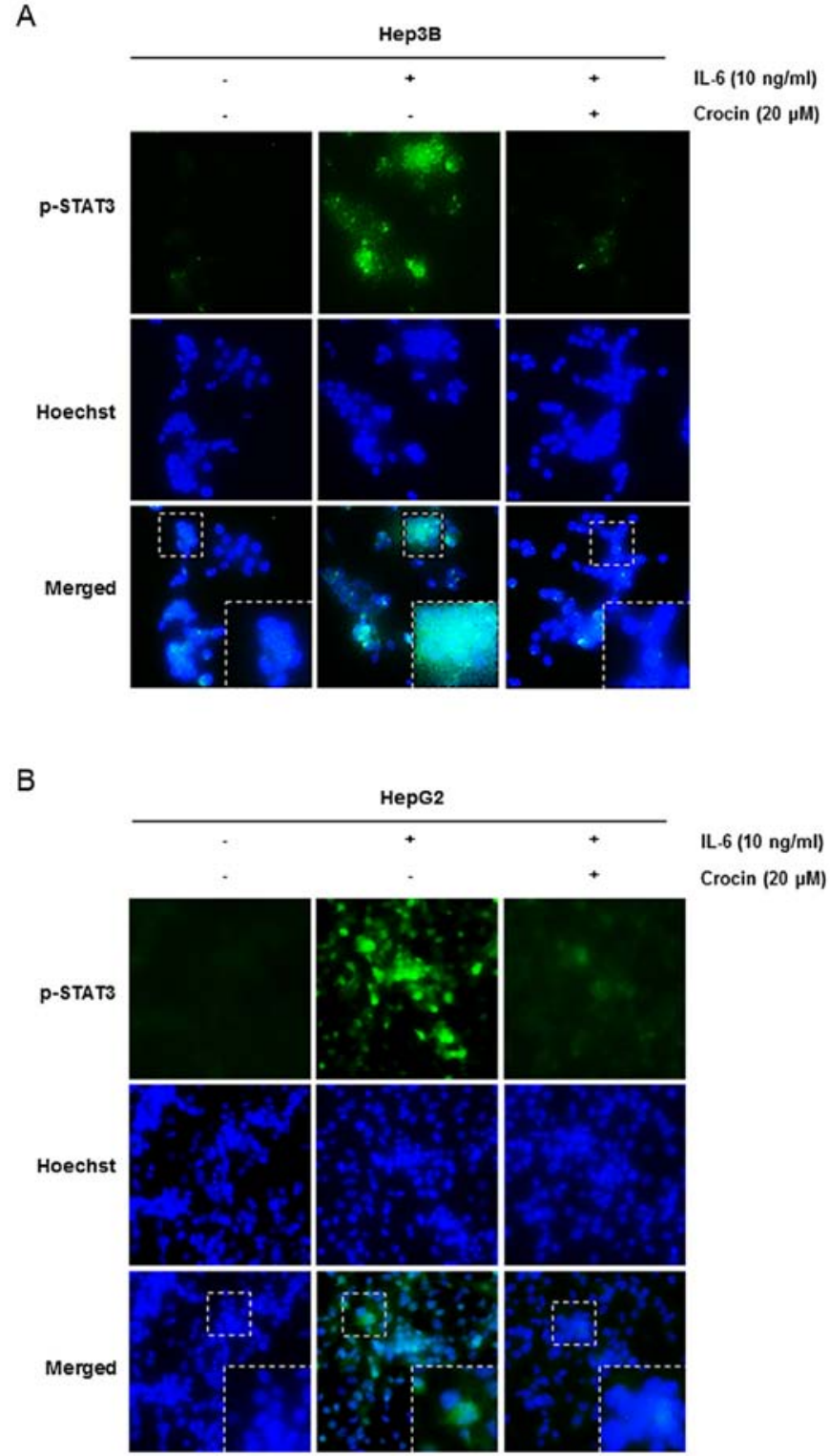

Figure 3. Crocin depletes the nuclear pool of STAT3 in IL-6-stimulated liver cancer cells. (A) Hep3B and (B) HepG2 cells were incubated with or without $20 \mu \mathrm{M}$ crocin for $24 \mathrm{~h}$ and then stimulated with IL-6 $(10 \mathrm{ng} / \mathrm{ml})$ for $60 \mathrm{~min}$. The intracellular distribution of STAT3 was analyzed by immune fluorescence. The cells were fixed and permeabilized STAT3 (green) was immune stained with rabbit anti-STAT3 followed by FITC-conjugated secondary antibodies and nuclei (blue) were stained with Hoechst 33342. The third panels display the merged images of the first and second panels.

Hep3B (Fig. 3A) and HepG2 (Fig. 3B) cells. Therefore, these results indicated that inhibition of STAT3 phosphorylation by crocin impaired STAT3 transcriptional function by blocking nuclear translocation.

Pervanadate reverses crocin-mediated inhibition of STAT3 phosphorylation in IL-6-stimulated Hep3B cells. Protein tyrosine phosphatases (PTPs) have been considered to be related to the STAT3 signaling pathway (29). Therefore, we investigated whether PTPs were involved in blockade of STAT3 signaling by crocin in Hep3B cells. Sodium pervanadate (a broad-acting tyrosine phosphatase inhibitor) reversed crocin-mediated inhibition of STAT3 activation induced by IL-6 (Fig. 4A), 
A

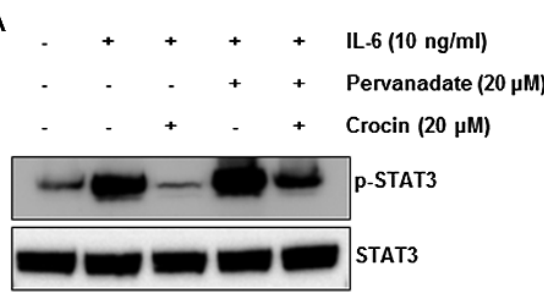

C

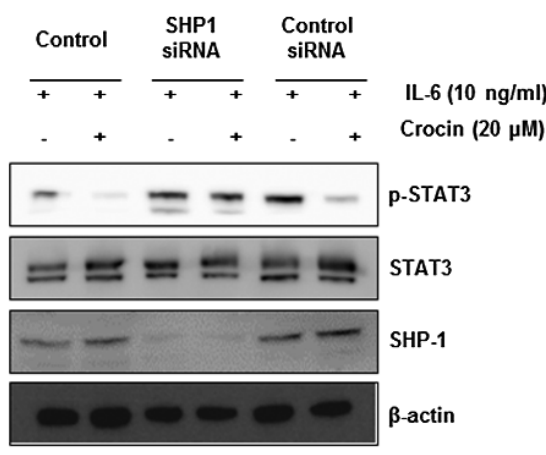

B

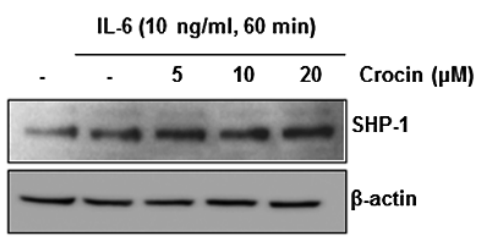

D

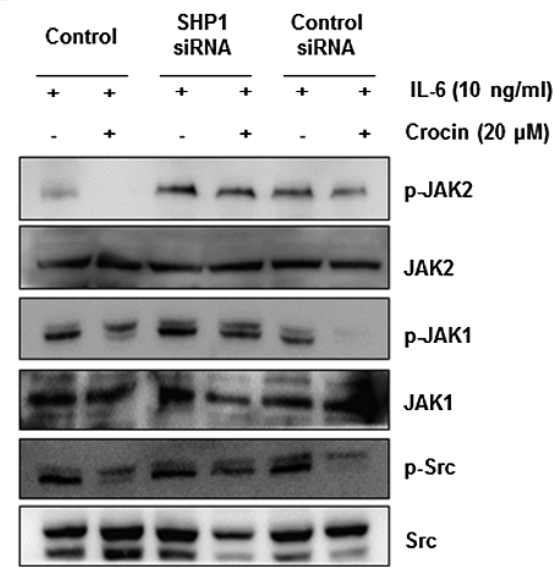

Figure 4. Crocin induces the expression of SHP-1 protein in IL-6-stimulated Hep3B cells. (A) Pervanadate reversed crocin-mediated inhibition of STAT3 phosphorylation in IL-6-stimulated Hep3B cells. The cells were treated with pervanadate or crocin for $24 \mathrm{~h}$, and then stimulated with IL-6 for 60 min. The phospho-STAT3 was detected by western blotting, and the same blots were stripped and reprobed with STAT3 antibodies. (B) Crocin induced the expression of the SHP-1 protein. Hep3B cells were treated with 0, 5, 10 and $20 \mu \mathrm{M}$ crocin for $24 \mathrm{~h}$ and then stimulated with IL-6 (10 ng/ml). After whole-cell extracts were prepared, SHP-1was detected by western blotting. The same blots were stripped and reprobed with the $\beta$-actin antibody to ascertain equal protein loading. (C and D) Hep3B cells were transfected with either SHP-1 siRNA or scrambled siRNA (50 nM). After $24 \mathrm{~h}$, the cells were treated with or without $20 \mu \mathrm{M}$ crocin for $24 \mathrm{~h}$ and then stimulated with IL-6 $(10 \mathrm{ng} / \mathrm{ml})$ for $60 \mathrm{~min}$. Then, whole-cell extracts were subjected to western blot analysis for SHP-1, p-STAT3, p-JAK1, p-JAK2 and p-Src. The same blots were stripped and reprobed with $\beta$-actin, STAT3, JAK1, JAK2 and Src antibodies to ascertain equal protein loading.

indicating that the inhibitory effect of crocin was related to at least one tyrosine phosphatase.

Since previous studies have shown that various natural products such as pectolinarigenin (30), capsazepine (31) and ginkgetin (32) dephosphorylate STAT3 through SHP-1 activation, we observed the protein level of SHP-1 in IL-6-stimulated Hep3B cells after crocin exposure. We determined that crocin increased SHP-1 expression in a dose-dependent manner (Fig. 4B). This result revealed that SHP-1 plays an important role in crocin-mediated inhibition of STAT3 activity.

Suppression of SHP-1 by a specific siRNA reverses crocinmediated inhibition of STAT3 phosphorylation. To confirm that dephosphorylation of STAT3 by crocin was due to SHP-1, a siRNA against SHP-1 was transfected into IL-6stimulated Hep3B cells after treatment with or without crocin. The results revealed that dephosphorylation of STAT3 by crocin was confirmed in the control group and the scrambled-siRNA transfected group. However, STAT3 dephosphorylation was recovered in the SHP-1-siRNA transfected group (Fig. 4C). These results corroborated our earlier evidence on the critical role of SHP-1 in suppression of STAT3 phosphorylation by crocin. SHP-1 gene silencing with siRNA did not suppress activation of JAK1, JAK2 and Src in IL-6-stimulated Hep3B cells (Fig. 4D). These data indicated that SHP-1 has a critical role in the inhibition of STAT3 signaling by crocin.
Crocin downregulates gene expression related to cell proliferation, survival, apoptosis, and invasion in IL-6-stimulated liver cancer cells. Cyclin D1 is required for cell proliferation and transition from G1 to S phase of the cell cycle and is regulated by STAT3. We revealed that crocin treatment suppressed the expression of cyclin D1 in a time-dependent manner in IL-6-stimulated liver cancer cells. In addition, there are many studies suggesting that STAT3 activation is associated with the expression of gene products involved in cancer metastasis (CXCR4) (33-35) and angiogenesis (VEGF) (36). Based on this, we observed that crocin treatment suppressed the expression of CXCR4 and VEGF in IL-6-stimulated liver cancer cells in both cell lines. Furthermore, STAT3 activation also regulates the expression of various gene products related to apoptosis, including Bcl-2, Bcl-xL, and surviving (6). To identify the role of crocin in apoptotic cell death, we performed western blot analysis of anti-apoptotic proteins, Bcl-2 and survivin. The results revealed that crocin significantly decreased the level of Bcl-2 and survivin and increased the level of BAX as shown in Fig. 5A. Based on these results, it was determined that crocin had an effect on antiproliferation, apoptosis and blockade of invasion in IL-6-stimulated liver cancer cells.

Crocin activates caspase-9, -3 and PARP cleavage. We also investigated whether suppression of STAT3 activation by crocin led to apoptosis. Cells were treated with $20 \mu \mathrm{M}$ of crocin for different time-points and then examined for 

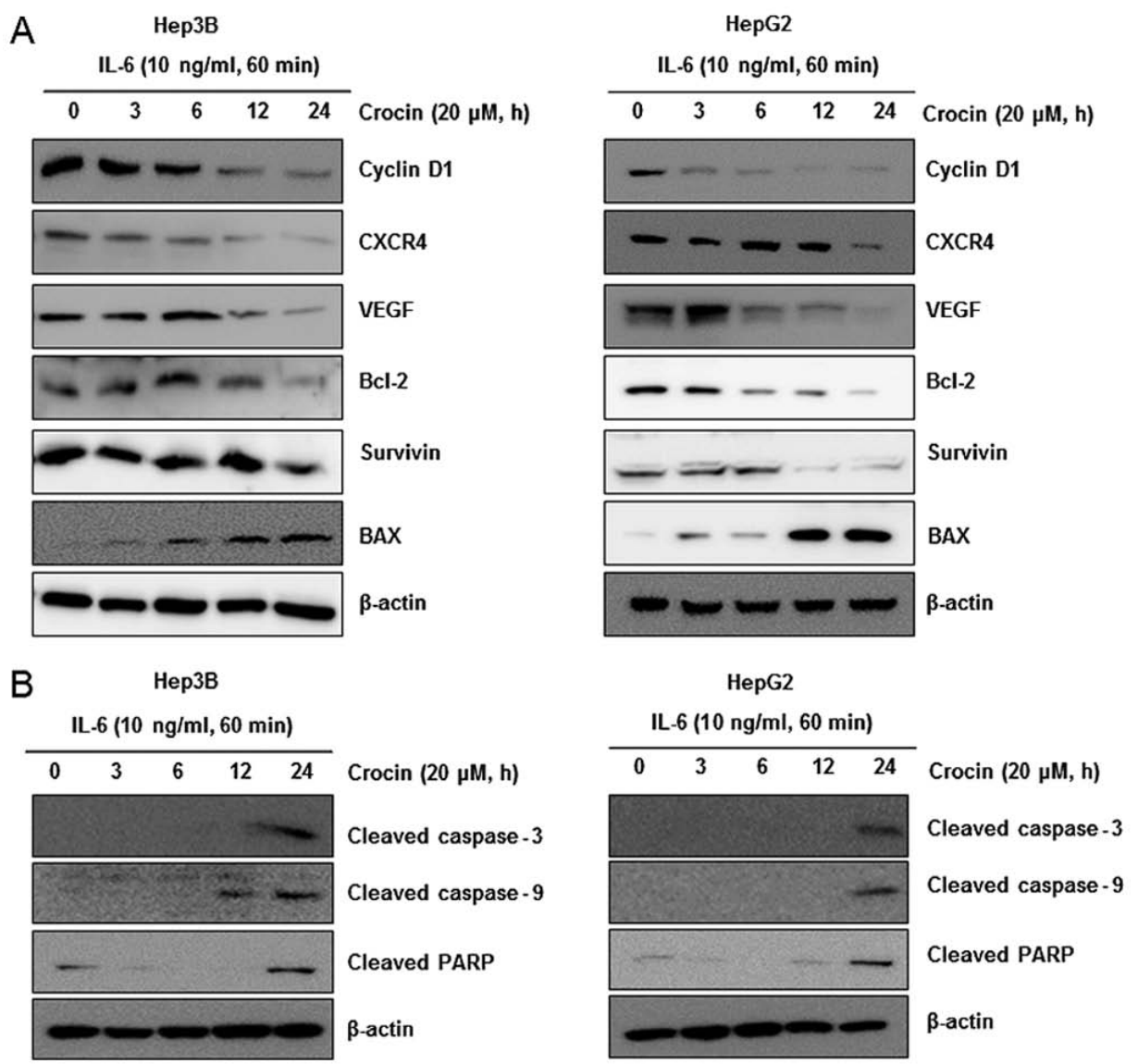

Figure 5. Crocin downregulates gene expression related to cell survival, proliferation, apoptosis, and invasion in IL-6-stimulated liver cancer cells. (A) Crocin suppresses STAT3-regulated gene products involved in survival, proliferation, apoptosis and invasion. Hep3B and HepG2 cells (1x10 $/ \mathrm{ml})$ were treated with $20 \mu \mathrm{M}$ crocin for indicated time-points and then stimulated with IL-6 $(10 \mathrm{ng} / \mathrm{ml})$. After whole-cell extracts were prepared and $30 \mu \mathrm{g}$ proteins of those extracts were resolved on $12 \%$ SDS-PAGE, the membranes were probed against cyclin D1, CXCR4, VEGF, Bcl-2, survivin and BAX antibodies. The same blots were stripped and reprobed with the $\beta$-actin antibody to ascertain equal protein loading. (B) Hep3B (left panel) and HepG2 (right panel) cells $\left(1 \times 10^{6} / \mathrm{ml}\right)$ were treated with $20 \mu \mathrm{M}$ crocin for indicated time-points and then stimulated IL-6 $(10 \mathrm{ng} / \mathrm{ml})$. After whole-cell extracts were prepared and $30 \mu \mathrm{g}$ proteins of those extracts were resolved on 8 or $12 \%$ SDS-PAGE, the membranes were probed against cleaved caspase-3, cleaved caspase- 9 and cleaved PARP antibodies. The same blots were stripped and reprobed with the $\beta$-actin antibody to ascertain equal protein loading.

activation of caspases by western blotting using specific antibodies. We observed cleavage of caspase- 9 , caspase- 3 and PARP at $20 \mu \mathrm{M}$ of crocin for $24 \mathrm{~h}$ (Fig. 5B). Finally, we also determined that cells exposed to crocin were TUNEL-positive, further corroborating with evidence the initiation of apoptosis in these cells. These results clearly revealed that crocin induced caspase-dependent apoptosis in IL-6-stimulated Hep3B (Fig. 6A) and HepG2 (Fig. 6B).

\section{Discussion}

STAT3 activation is closely involved in a wide range of tumor types such as carcinoma, sarcoma, lymphoma and leukemia $(6,37)$ and pharmacological inhibition of STAT3 has shown its potential in cancer prevention and treatment. In addition, interleukin-6 (IL-6) has been reported to be closely related to STAT3 activity. The purpose of this study was to examine whether crocin, derived from Crocus sativus L. (saffron), exerted its anticancer effects through abrogation of the STAT3 signaling pathway induced by IL-6 in liver cancer cells. We first investigated whether crocin inhibited STAT3 activation induced by IL-6 in Hep3B and HepG2 cells. The results revealed that crocin inhibited STAT3 activation and this was related to inactivation of upstream kinases and induction of protein tyrosine phosphatase (PTP). Moreover, blockade of STAT3 activation led to suppression of gene expression regulated by STAT3. Recent studies have revealed that STAT3, which plays an important role in carcinogenesis, may contribute to designing novel targeted therapies $(30,31)$. We found that crocin suppressed IL-6-induced STAT3 activation and STAT3-DNA binding in liver cancer cells. Phosphorylation of STAT3 is mediated through activation of non-receptor protein tyrosine kinases, including Janus-like kinases (JAK)-1, -2, TYK2, and c-Src kinasec(38). Thus, we examined whether crocin had an inhibitory effect on these kinases that have demonstrated potential in the STAT3 activation pathway. Notably, we observed that crocin inhibited activation of JAK-1, JAK-2 and Src in IL-6-stimulated Hep3B cells. The SH2 domain-containing phosphatase 1 (SHP-1) is a non-receptor PTP that has been revealed in hematopoietic cells $(39,40)$. Specifically, SHP-1 has tumor-suppression potential due to negative regulation of STAT3 signaling during tumor progression $(41,42)$. We revealed that crocin treatment significantly increased the expression of SHP-1 and this was correlated with a decrease in p-STAT3 expression. We also demonstrated that crocin had no effect on suppression of 
A
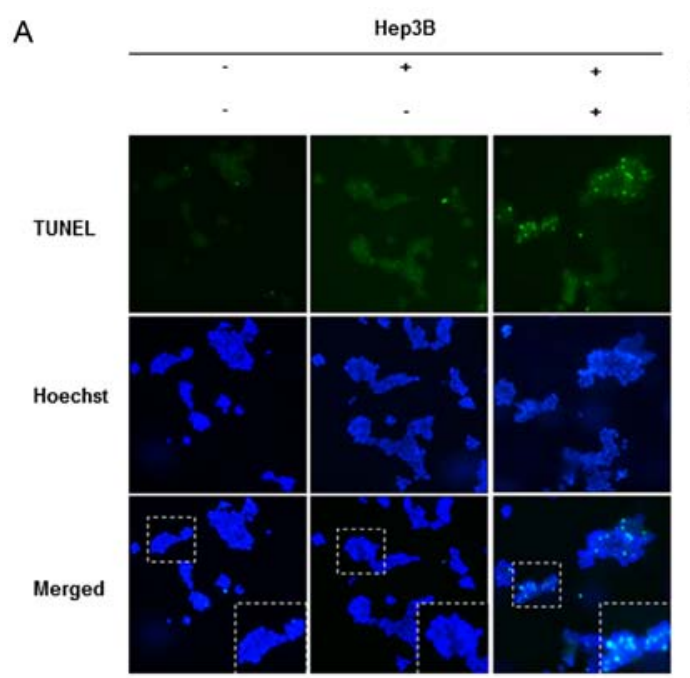

B

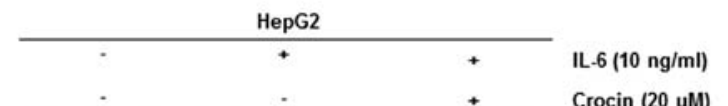

TUNEL
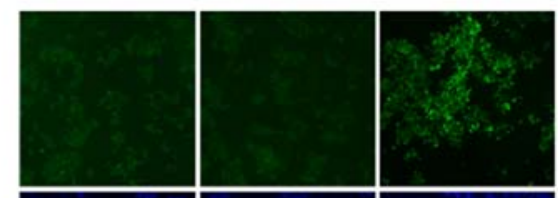

Hoechst
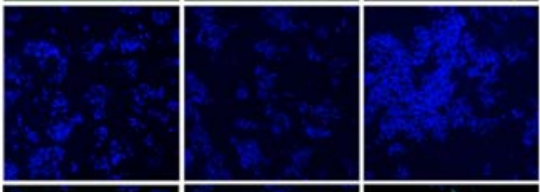

Merged
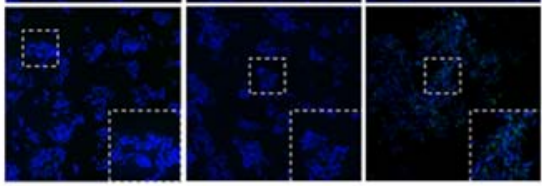

Figure 6. Crocin induces apoptotic progression in Hep3B and HepG2 cells. TUNEL-positive cells were significantly induced in the IL-6-stimulated (A) Hep3B and (B) HepG2 cells by crocin administration.

p-STAT3 when the cells were transfected with SHP-1-siRNA. Notably, our present findings revealed that the tyrosine phosphatase SHP-1, which is upregulated by crocin, plays a critical role in suppression of the STAT3 signaling pathway in IL-6stimulated liver cancer cells.

STAT3 activation, which results in encoding anti-apoptotic proteins and proliferation-associated proteins and leads to decreased cell death, is strongly associated with the development of various types of cancer $(43,44)$. Apoptosis is the process of programmed cell death characterized by a series of morphological changes, including plasma and nuclear membrane blebbing, cell shrinkage, and caspase activation. In the present study, we detected that crocin significantly promoted the activation of caspase- 3 and caspase- 9 along with enhanced cleavage of PARP, indicating that crocin may exert its anticancer activity via enabling induction of apoptosis. In addition, STAT3 has been confirmed to be involved in the regulation of anti-apoptotic proteins and the inhibitor of apoptosis protein (IAP) family (45). We also demonstrated that crocin decreased the expression levels of Bcl-2 and survivin, suggesting that crocin induces apoptosis in Hep3B and HepG2 cells stimulated with IL-6. Various natural drugs have been reported to inhibit cyclin D1, VEGF and CXCR4, which are regulated by STAT3. For example, quercetin inhibited the IL-6/STAT3 signaling pathway and inhibited growth and migration of glioblastoma cells (46). Plumbagin downregulated cyclin D1 and VEGF expression through STAT3 regulation (47), and sinomenine inhibited the invasion and metastasis through inhibition of the CXCR4-STAT3 pathway (48). As observed in these studies, crocin ameliorated the expression of genes related to cell proliferation and invasion, as evidenced by decreased expression of cyclin D1, VEGF and CXCR4.

In conclusion, the present study provides evidence that crocin has the potential for anticancer activity through inhibition of the IL-6/STAT3 signaling pathway, especially in liver cancer. However, further research using clinical animal models is warranted to realize all the potential of this molecule as an anticancer drug.

\section{Acknowledgements}

This study was supported by the Basic Science Research Program through the National Research Foundation of Korea (NRF) funded by the Ministry of Education (NRF-2016R1A6A1A03011325) and by the Ministry of Science, ICT \& Future Planning (NRF-2016R1A1A1A05921696).

\section{Competing interests}

The authors declare that they have no competing interests.

\section{References}

1. Jemal A, Bray F, Center MM, Ferlay J, Ward E and Forman D: Global cancer statistics. CA Cancer J Clin 61: 69-90, 2011.

2. Villanueva A, Hernandez-Gea V and Llovet JM: Medical therapies for hepatocellular carcinoma: A critical view of the evidence. Nat Rev Gastroenterol Hepatol 10: 34-42, 2013.

3. Zhao C, Wang W, Yu W, Jou D, Wang Y, Ma H, Xiao H, Qin H, Zhang C, Lü J, et al: A novel small molecule STAT3 inhibitor, LY5, inhibits cell viability, colony formation, and migration of colon and liver cancer cells. Oncotarget 7: 12917-12926, 2016.

4. Zhang Z, Mao H, Du X, Zhu J, Xu Y, Wang S, Xu X, Ji P, Yu Y, Cao B, et al: A novel small molecule agent displays potent anti-myeloma activity by inhibiting the JAK2-STAT3 signaling pathway. Oncotarget 7: 9296-9308, 2016.

5. Lanton T, Shriki A, Nechemia-Arbely Y, et al: IL6-Dependent genomic instability heralds accelerated carcinogenesis following liver regeneration on a background of chronic hepatitis. Hepatology 65: 1600-1611, 2017.

6. Aggarwal BB, Sethi G, Ahn KS, Sandur SK, Pandey MK, Kunnumakkara AB, Sung B and Ichikawa H: Targeting signaltransducer-and-activator-of-transcription-3 for prevention and therapy of cancer: modern target but ancient solution. Ann N Y Acad Sci 1091: 151-169, 2006.

7. Zhao H, Guo Y, Li S, Han R, Ying J, Zhu H, Wang Y, Yin L, Han Y, Sun L, et al: A novel anti-cancer agent Icaritin suppresses hepatocellular carcinoma initiation and malignant growth through the IL-6/Jak2/Stat3 pathway. Oncotarget 6: 31927-31943, 2015.

8. Khorasany AR and Hosseinzadeh $\mathrm{H}$ : Therapeutic effects of saffron (Crocus sativus L.) in digestive disorders: a review. Iran J Basic Med Sci 19: 455-469, 2016.

9. Kim SH, Lee JM, Kim SC, Park CB and Lee PC: Proposed cytotoxic mechanisms of the saffron carotenoids crocin and crocetin on cancer cell lines. Biochem Cell Biol 92: 105-111, 2014.

10. Asdaq SM and Inamdar MN: Potential of Crocus sativus (saffron) and its constituent, crocin, as hypolipidemic and antioxidant in rats. Appl Biochem Biotechnol 162: 358-372, 2010.

11. Ordoudi SA, Befani CD, Nenadis N, Koliakos GG and Tsimidou MZ: Further examination of antiradical properties of Crocus sativus stigmas extract rich in crocins. J Agric Food Chem 57: 3080-3086, 2009. 
12. Xu GL, Yu SQ, Gong ZN and Zhang SQ: Study of the effect of crocin on rat experimental hyperlipemia and the underlying mechanisms. Zhongguo Zhong Yao Za Zhi 30: 369-372, 2005 (In Chinese)

13. Wang Y, Han T, Zhu Y, Zheng CJ, Ming QL, Rahman K and Qin LP: Antidepressant properties of bioactive fractions from the extract of Crocus sativus L. J Nat Med 64: 24-30, 2010.

14. Nam KN, Park YM, Jung HJ, Lee JY, Min BD, Park SU, Jung WS, Cho KH, Park JH, Kang I, et al: Anti-inflammatory effects of crocin and crocetin in rat brain microglial cells. Eur J Pharmacol 648: 110-116, 2010

15. Xu GL, Li G, Ma HP, Zhong H, Liu F and Ao GZ: Preventive effect of crocin in inflamed animals and in LPS-challenged RAW 264.7 cells. J Agric Food Chem 57: 8325-8330, 2009

16. Xu HJ, Zhong R, Zhao YX, Li XR, Lu Y, Song AQ, Pang XY, Yao RY and Sun LR: Proliferative inhibition and apoptotic induction effects of crocin on human leukemia HL-60 cells and their mechanisms. Zhongguo Shi Yan Xue Ye Xue Za Zhi 18 887-892, 2010 (In Chinese).

17. Aung HH, Wang CZ, Ni M, Fishbein A, Mehendale SR, Xie JT, Shoyama CY and Yuan CS: Crocin from Crocus sativus possesses significant anti-proliferation effects on human colorectal cancer cells. Exp Oncol 29: 175-180, 2007.

18. Chryssanthi DG, Lamari FN, Iatrou G, Pylara A, Karamanos NK and Cordopatis P: Inhibition of breast cancer cell proliferation by style constituents of different Crocus species. Anticancer Res 27 : 357-362, 2007.

19. Zhao P, Luo CL, Wu XH, Hu HB, Lv CF and Ji HY: Proliferation apoptotic influence of crocin on human bladder cancer T24 cell line. Zhongguo Zhong Yao Za Zhi 33: 1869-1873, 2008 (In Chinese)

20. Mashmoul M, Azlan A, Mohtarrudin N, Mohd Yusof BN, Khaza'ai H, Khoo HE, Farzadnia $M$ and Boroushaki MT: Protective effects of saffron extract and crocin supplementation on fatty liver tissue of high-fat diet-induced obese rats. BMC Complement Altern Med 16: 401, 2016.

21. Deng J, Grande F and Neamati N: Small molecule inhibitors of Stat3 signaling pathway. Curr Cancer Drug Targets 7: 91-107, 2007.

22. Yu D, Ye T, Xiang Y, Shi Z, Zhang J, Lou B, Zhang F, Chen B and Zhou M: Quercetin inhibits epithelial-mesenchymal transition, decreases invasiveness and metastasis, and reverses IL-6 induced epithelial-mesenchymal transition, expression of MMP by inhibiting STAT3 signaling in pancreatic cancer cells. Onco Targets Ther 10: 4719-4729, 2017.

23. Wang L, Cao L, Wang H, Liu B, Zhang Q, Meng Z, Wu X, Zhou Q and $\mathrm{Xu} \mathrm{K}$ : Cancer-associated fibroblasts enhance metastatic potential of lung cancer cells through IL-6/STAT3 signaling pathway. Oncotarget 8: 76116-76128, 2017.

24. Zhao X, Huang L, Xu W, Chen X, Shen Y, Zeng W and Chen X Physapubescin B inhibits tumorgenesis and circumvents taxol resistance of ovarian cancer cells through STAT3 signaling. Oncotarget 8: 70130-70141, 2017.

25. Zhang YX, Yan L, Liu GY, Chen WJ, Gong WH and Yu JM: Inhibition of janus kinase 2 by compound AG490 suppresses the proliferation of MDA-MB-231 cells via up-regulating SARI (suppressor of AP-1, regulated by IFN). Iran J Basic Med Sci 18: 599-603, 2015

26. Fan LC, Teng HW, Shiau CW, Tai WT, Hung MH, Yang SH, Jiang JK and Chen KF: Pharmacological targeting SHP-1-STAT3 signaling is a promising therapeutic approach for the treatment of colorectal cancer. Neoplasia 17: 687-696, 2015.

27. Yu CL, Meyer DJ, Campbell GS, Larner AC, Carter-Su C, Schwartz $\mathrm{J}$ and Jove R: Enhanced DNA-binding activity of a Stat3-related protein in cells transformed by the Src oncoprotein. Science 269: 81-83, 1995.

28. Kim BH, Won C, Lee YH, Choi JS, Noh KH, Han S, Lee H, Lee CS, Lee DS, Ye SK and Kim MH: Sophoraflavanone G induces apoptosis of human cancer cells by targeting upstream signals of STATs. Biochem Pharmacol 86: 950-959, 2013.

29. Darnell JE Jr: STATs and gene regulation. Science 277: 1630-1635, 1997.

30. Zhang T, Li S, Li J, Yin F, Hua Y, Wang Z, Lin B, Wang H, Zou D, Zhou Z, et al: Natural product pectolinarigenin inhibits osteosarcoma growth and metastasis via SHP-1-mediated STAT3 signaling inhibition. Cell Death Dis 7: e2421, 2016.
31. Lee JH, Kim C, Baek SH, Ko JH, Lee SG, Yang WM, Um JY, Sethi G and Ahn KS: Capsazepine inhibits JAK/STAT3 signaling, tumor growth, and cell survival in prostate cancer. Oncotarget 8: 17700-17711, 2017.

32. Baek SH, Lee JH, Ko JH, Lee H, Nam D, Lee SG, Yang WM, Um JY, Lee J, Kim SH, et al: Ginkgetin blocks constitutive STAT3 activation and induces apoptosis through induction of SHP-1 and PTEN tyrosine phosphatases. Phytother Res 30: 567-576, 2016.

33. Liu X, Xiao Q, Bai X, Yu Z, Sun M, Zhao H, Mi X, Wang E, Yao W, Jin F, et al: Activation of STAT3 is involved in malignancy mediated by CXCL12-CXCR4 signaling in human breast cancer. Oncol Rep 32: 2760-2768, 2014.

34. Pfeiffer M, Hartmann TN, Leick M, Catusse J, Schmitt-Graeff A and Burger M: Alternative implication of CXCR4 in JAK2/STAT3 activation in small cell lung cancer. Br J Cancer 100: 1949-1956, 2009.

35. Tang Y, Guo Q, Zhi Y, Jin X, Xia B, Guo S, Tian C and Zhang Y: Role of CXCR4/STAT3 in mesenchymal stromal cell-mediated drug resistance of acute leukemia cells. Zhonghua Xue Ye Xue Za Zhi 37: 119-123, 2016 (In Chinese).

36. Yahata Y, Shirakata Y, Tokumaru S, Yamasaki K, Sayama K, Hanakawa Y, Detmar M and Hashimoto K: Nuclear translocation of phosphorylated STAT3 is essential for vascular endothelial growth factor-induced human dermal microvascular endothelial cell migration and tube formation. J Biol Chem 278: 40026-40031, 2003.

37. Pandey MK, Sung B and Aggarwal BB: Betulinic acid suppresses STAT3 activation pathway through induction of protein tyrosine phosphatase SHP-1 in human multiple myeloma cells. Int J Cancer 127: 282-292, 2010.

38. Yu H, Pardoll D and Jove R: STATs in cancer inflammation and immunity: A leading role for STAT3. Nat Rev Cancer 9: 798-809, 2009.

39. Banville D, Stocco R and Shen SH: Human protein tyrosine phosphatase 1C (PTPN6) gene structure: Alternate promoter usage and exon skipping generate multiple transcripts. Genomics 27: 165-173, 1995.

40. Tsui HW, Hasselblatt K, Martin A, Mok SC and Tsui FW: Molecular mechanisms underlying SHP-1 gene expression. Eur J Biochem 269: 3057-3064, 2002.

41. Wu C, Sun M, Liu L and Zhou GW: The function of the protein tyrosine phosphatase SHP-1 in cancer. Gene 306: 1-12, 2003

42. Wu C, Guan Q, Wang Y, Zhao ZJ and Zhou GW: SHP-1 suppresses cancer cell growth by promoting degradation of JAK kinases. J Cell Biochem 90: 1026-1037, 2003.

43. Morikawa T, Baba Y, Yamauchi M, Kuchiba A, Nosho K, Shima K, Tanaka N, Huttenhower C, Frank DA, Fuchs CS and Ogino S: STAT3 expression, molecular features, inflammation patterns, and prognosis in a database of 724 colorectal cancers. Clin Cancer Res 17: 1452-1462, 2011.

44. Wang SW and Sun YM: The IL-6/JAK/STAT3 pathway: Potential therapeutic strategies in treating colorectal cancer (Review). Int J Oncol 44: 1032-1040, 2014.

45. Iwamaru A, Szymanski S, Iwado E, Aoki H, Yokoyama T, Fokt I, Hess K, Conrad C, Madden T, Sawaya R, et al: A novel inhibitor of the STAT3 pathway induces apoptosis in malignant glioma cells both in vitro and in vivo. Oncogene 26: 2435-2444, 2007.

46. Michaud-Levesque J, Bousquet-Gagnon N and Beliveau R: Quercetin abrogates IL-6/STAT3 signaling and inhibits glioblastoma cell line growth and migration. Exp Cell Res 318: 925-935, 2012.

47. Sandur SK, Pandey MK, Sung B and Aggarwal BB: 5-hydroxy2-methyl-1,4-naphthoquinone, a vitamin K3 analogue, suppresses STAT3 activation pathway through induction of protein tyrosine phosphatase, SHP-1: Potential role in chemosensitization. Mol Cancer Res 8: 107-118, 2010.

48. Xie T, Ren HY, Lin HQ, Mao JP, Zhu T, Wang SD and Ye ZM: Sinomenine prevents metastasis of human osteosarcoma cells via $\mathrm{S}$ phase arrest and suppression of tumor-related neovascularization and osteolysis through the CXCR4-STAT3 pathway. Int J Oncol 48: 2098-2112, 2016. 